\title{
Steroid cell tumour of ovary: A rare clinicopathologic entity
}

\author{
Shashikala Karanth ${ }^{1}$, A.P. Roshini², Sheela C N ${ }^{3, *}$, Rajalakshmi T ${ }^{4}$ \\ ${ }^{\mathbf{1}}$ Associate Professor, ${ }^{2}$ Final Year MBBS, ${ }^{3}$ Professor and Head, ${ }^{4}$ Professor, Dept. of Obstetrics \& Gynaecology, St. John's \\ Medical College and Hospital, Bengaluru, Karnataka, India
}

*Corresponding Author:

Email: drsheela1960@gmail.com

\begin{abstract}
'Steroid cell tumors not otherwise specified (SCT-NOS)' are rare sex cord-stromal tumors of the ovary with malignant potential, accounting for less than $0.1 \%$ of all ovarian tumors with the highest incidence in premenopausal women. These tumours represent a unique cause of female virilization. We present a case of a 48-year-old post-menopausal lady with bleeding per vagina and a 2-year history of truncal obesity and hirsutism. Past history was unremarkable. Examination revealed feature of obesity, virilization and clitoromegaly. Serum testosterone was $3.62 \mu \mathrm{g} / \mathrm{L} \&$ CA-125 was 40.67. CECT gave the evidence of adnexal tumor, but histopathology confirmed SCT-NOS. Total abdominal hysterectomy(TAH) and bilateral salpingoophorectomy(BSO) was performed. Post-operative period was uneventful. This case is being reported for its rarity of occurrence and challenges faced in diagnosis of this case.
\end{abstract}

Keywords: Virilization, Steroid cell tumour of the ovary, Not otherwise specified (NOS).

\section{Introduction}

Different cells of origin account for the diverse histo-pathologies of ovarian tumours. A majority, 90\%, are epithelial in origin. 2-6.5 cases are detected per $1,00,000$ women per year in Asian countries. ${ }^{1}$ These tumors present in child bearing age group, $3^{\text {rd }} \& 4^{\text {th }}$ decade with symptoms of androgen excess, manifested by virilization and extremely elevated testosterone levels. ${ }^{2}$ In cases of unexplained hirsutism, ovarian and adrenal tumour association should be ruled out and occult malignancies must be investigated for. We here by present a case report of 48 years old postmenopausal lady with signs of androgen excess and postmenopausal bleeding, which is finally proved to be a Steroid cell tumor- Not otherwise specified (SCTNOS).

\section{Case Report}

A 48 years old postmenopausal lady presented with hair growth over face and back for the last 2 years, bleeding per vaginum for 3 weeks and lower abdominal pain for 1 week. There was no history of recent weight gain, change in voice and no drug intake. There was no history of diabetes mellitus, hypertension or thyroid in the past. She had attained menopause 3 years back and was not on any Hormone Replacement Therapy (HRT). There was no history of gynecological or breast malignancies in the family

Examination revealed a normotensive female with acanthosis nigricans and hoarseness of voice. Her BMI was $34.81 \mathrm{~kg} / \mathrm{m} 2$ with a weight of $91 \mathrm{kgs}$ and height of $167 \mathrm{~cm}$. Breast examination was normal. She had hirsutism and according to Ferriman Gallwey Scoring (FGS), score was 36/36. Systemic examination was normal. Local inspection revealed clitoromegaly and per speculum examination revealed a normal cervix.
Vaginal examination revealed no abnormality. Per rectal examination revealed a free mucosa.

Patient was evaluated for post-menopausal bleeding and features of androgen excess. Routine investigations were normal. Pelvic Ultrasonography (USG) showed normal uterus and ovaries. Abdominal USG was also normal. PAP smear and hysteroscopy was normal. Histopathology of endometrial biopsy showed cystically dilated secretory glands set in a predecidualised stroma. DHEAS was $117 \mu \mathrm{g} / \mathrm{dL}$ and Serum Testosterone was elevated to $6.3 \mathrm{ng} / \mathrm{mL}$ (normal 0.1 - $1.2 \mathrm{ng} / \mathrm{mL})$. CA-125 was $40.67 \mathrm{IU} / \mathrm{ml}$, LH was $0.07 \mathrm{MIU} / \mathrm{mL}$ (normal $15.9-54 \mathrm{MIU} / \mathrm{mL}$ ) and $\mathrm{FSH}$ was $0.37 \mathrm{MIU} / \mathrm{mL} \quad$ (normal 23.0-116.3MIU/mL). Dexamethasone suppression test suppressed cortisol from 19.29 to $0.81 \mu \mathrm{g} / \mathrm{mL}$. Since USG did not reveal any cause, CECT was done to look for adnexal and adrenal pathology. CECT showed a dumb-bell shaped lesion with central constriction showing separate intensities is seen in the left adnexa adjacent to left ovary (Fig. 1).

Staging laparotomy was performed, peritoneal fluid was sent for cytological analysis. There was a $4 \times 4 \mathrm{~cm}$ friable, yellow mass on the left ovary (Fig. 2). Left salpingo-oophorectomy was done and sent for frozen section (Fig. 2), which revealed Steroid Cell tumour of benign nature characterized by polygonal cells with abundant clear to vacuolated cytoplasm with no evidence of necrosis, haemorrhage or increased mitoses (Fig. 3). The oil red $\mathrm{O}$ stain showed the abundant lipid content in the cells, confirming the diagnosis. (Fig. 3). Total abdominal hysterectomy and right salpingo-oophorectomy was done. Abdominal organs and omentum were normal. Post-operative period was uneventful and she was discharged on day 6 . Final histopathology report was concordant and there were no malignant cells in peritoneal fluid. Her serum 
testosterone fell significantly after 1 week of surgery and she is doing well on follow up.

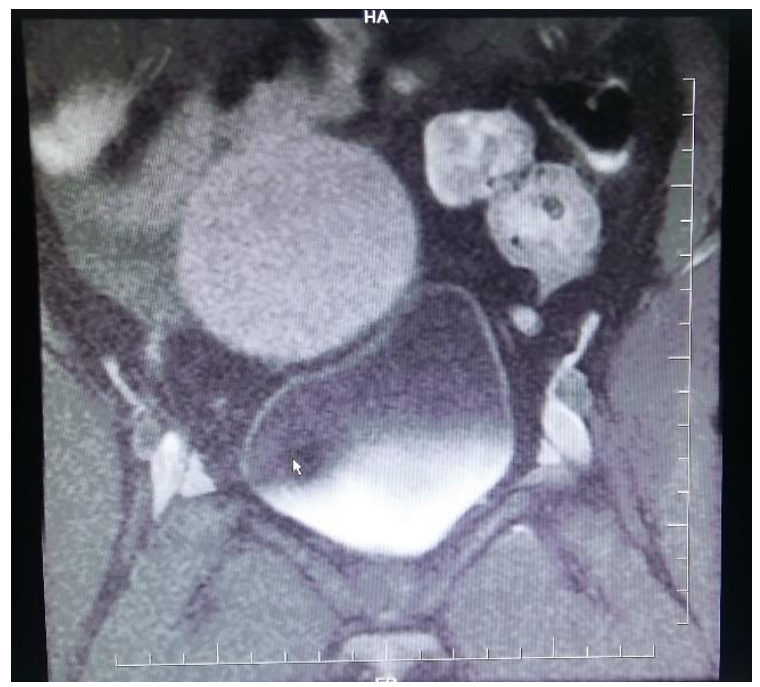

Fig. 1: CECT - dumb-bell lesion with central constriction in the Left adnexa adjacent to Left ovary
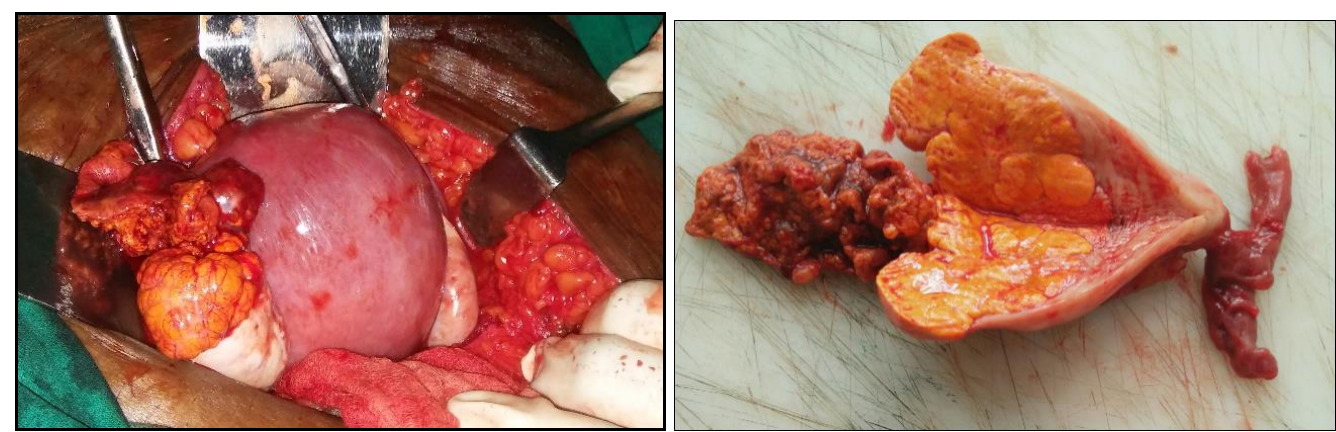

Fig. 2: Intra-operatively- left adnexal lesion adjacent to the left ovary; normal right ovary; Specimen/Cutsection $-4 \times 4 \mathrm{~cm}$, friable, unencapsulated, yellowish mass
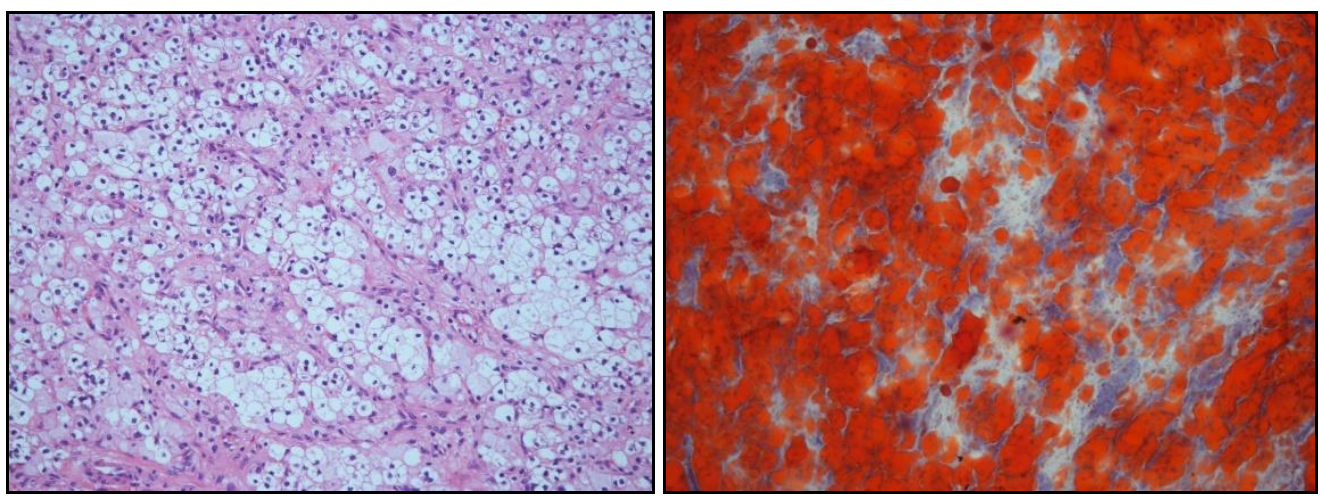

Fig. 3: Histopathology-polygonal, vacuolated clear cells arranged in nests; Oil red $O$ stain- abundant lipid content in the cells

\section{Discussion}

The term 'Steroid cell tumors not otherwise specified (SCT-NOS)' was $1^{\text {st }}$ used by Scully in 1979. These tumours account for $<0.1 \%$ of all ovarian tumours. ${ }^{1}$ Highest incidence is in the $3^{\text {rd }}-4^{\text {th }}$ decade. rarely it can occur in post-menopausal women and in young children. ${ }^{2}$ Steroid cell tumors are sex cord stromal tumours and are sub-classified into 3 typesstromal luteoma, Leydig cell tumour and not otherwise specified. SCT-NOS signifies that the cell lineage is not defined and thus, cannot be categorized as either stromal luteomas or Leydig cell tumors. ${ }^{2,3}$ These tumors represent a unique cause of female virilization. Approximately $56-77 \%$ of the cases are clinically associated with androgenic changes, such as hirsutism 
and virilization; 6-7\% of the cases are clinically associated with cushing's syndrome; and $25 \%$ of SCTNOS are non-functional. ${ }^{4}$ The clinical presentation is non-specific, including abdominal pain, distension and bloating. However, the more significant presentations are due to the hormonal activity and virilising properties of the tumour as it was in our case. A comprehensive workup, therefore, includes the evaluation of an adrenal and ovarian source of hyperandrogenism is required. NOS subtype accounts for $60 \%$ of steroid cell tumours. Majority of these tumours are benign; only $25 \%$ of them become malignant. ${ }^{5}$ Main stay of treatment in these tumours is surgery, depending on whether they are in the reproductive age group or not. For those who have completed child bearing, as was in our patient, TAH with BSO and complete staging is indicated. Adjuvant chemotherapy or radiation should be considered based on histopathology and survival staging but their therapeutic effect is poorly understood. ${ }^{6}$ Chemotherapy post-operatively, in those who show malignant features is still a controversy as there are no well-defined guidelines. PVB (cisplatin. Vincristine and bleomycin) or BEP (bleomycin, etoposide and cisplatin) are under trial currently. ${ }^{7}$

\section{Conclusion}

SCT-NOS have a challenging presentation and a high index of suspicion is required. Systematic evaluation of the patient is required to differentiate between an ovarian and adrenal tumour. CECT, in this case, gave the evidence of tumour; but a histopathology confirmed diagnosis.

\section{Source of Funding: None}

\section{Conflicts of Interest: None}

\section{References}

1. Scully RE. Ovarian tumors. A review. Am J Pathol. 1977;87:686-720.

2. Ranjeev Bhagat, Vijay Kumar Bodal, Nishit Gupta, Pooja Garg. Steroid Cell Tumour of Ovary - A Rare Case Report. J Clin Diagn Res. 2016 September; 10(9):ED06ED07.

3. Hayes MC, Scully RE: Ovarian steroid cell tumors (not otherwise specified): a clinicopathological analysis of 63 cases. Am J Surg Pathol 1987, 11:835-845.

4. Wei Jiang, Xiang Tao Fa, ng Fang, Shaofen Zhang, Congjian $\mathrm{Xu}$;; Benign and malignant ovarian steroid cell tumors, not otherwise specified: case studies, comparison, and review of the literature; J Ovarian Res;2013;6:53.

5. Paul Singh, Frank Deleon, and Ralph Anderson, "Steroid Cell Ovarian Neoplasm, Not Otherwise Specified: A Case Report and Review of the Literature," Case Reports in Obstetrics and Gynecology, vol. 2012, Article ID 253152, 3 pages, 2012.

6. Yi-Jou Tai, Wen-Chun Chang, Kuan-Ting Kuo, BorChing Sheu. Ovarian steroid cell tumor, not otherwise specified, with virilization symptoms. Taiwanese Journal of Obstetrics and Gynecology, 2014-06-01, Volume 53, Issue 2, Pages 260-262.

7. Reedy MB, Richards WE, Ueland F, Uy K, Lee EY, Bryant C, van Nagell JR Jr: Ovarian steroid cell tumors, not otherwise specified: a case report and literature review. Gynecol Oncol 1999, 75:293-297. 